\title{
The waves at the Mulberry Harbours
}

\author{
Zoe Jackson $^{1} \cdot$ Stephen Grey $^{2} \cdot$ Thomas A. A. Adcock $^{1}$ (D) Paul H. Taylor ${ }^{1,3}$. \\ Jean-Raymond Bidlot ${ }^{4}$
}

Received: 22 March 2017 / Accepted: 27 June 2017 / Published online: 12 July 2017

(C) The Author(s) 2017. This article is an open access publication

\begin{abstract}
The Mulberry Harbours were used during the Second World War as part of Operation Overlord, the invasion of northern Europe by the Allies in June 1944. This commenced with the D-Day landings on the Normandy beaches on 6th June. The harbours played an important role in the history of ocean engineering leading to the development of novel technology and new theory. A severe storm occurred soon after the harbours were deployed leading to the destruction of the American harbour and severe damage to the British one. In this paper, we analyse this storm using hindcast data from ECMWF and SWAN modelling. We find that the waves were significantly more severe at the American harbour than at the British one, which may partially explain why the latter experienced less damage. We also find that the usually quoted figure for the storm severity of 1 in 40 years is a reasonable estimate for a summer storm at these locations.
\end{abstract}

Keywords Mulberry Harbours · D-Day

Thomas A. A. Adcock

thomas.adcock@eng.ox.ac.uk

1 Department of Engineering Science, University of Oxford, Oxford, UK

2 HR Wallingford, Wallingford, UK

3 Faculty of Engineering, Computing and Mathematics, The University of Western Australia, 35 Stirling Highway, Crawley, WA 6009, Australia

4 European Centre for Medium-Range Weather Forecasts, Reading, UK

\section{Introduction}

The Mulberry Harbours were deployed to support the Allied invasion of Normandy during the Second World War as part of Operation Overlord. The Harbours are of obvious historical interest to those studying military history-perhaps less obvious is the important role they played, in some ways indirectly, in advancing coastal and ocean engineering (see for instance the introductions to Dean and Dalrymple 1991; Tucker and Pitt 2001; Reeve et al. 2004). Almost all the components of the harbour had novel features some of which have been used in later designs. As important, much theory was developed as part of the design process, or in consequence of problems with the harbours, bringing into the field many of the people who would go on to be major figures in the field to the study of wave mechanics. ${ }^{1}$

The focus of this paper is an analysis of the waves which occurred during the large storm which occurred a fortnight after D-Day. This paper is not an in-depth review of the design, construction and deployment of the harbours although a brief summary of these is given as background. The storm ruined one of the harbours and badly damaged the other. The storm led to serious supply problems for Operation Overlord at a crucial time in the invasion. This study analyses the magnitude of the waves during this storm and whether such a severe event should have been expected.

\section{Background}

This section presents a brief overview of the harbours. A detailed account is given in Hartcup (1977) with more

\footnotetext{
1 We note however that whilst the great Walter Munk was involved in wave prediction during the war (in North Africa and in the Pacific), he was not directly involved with the Mulberry Harbours themselves as is often suggested (Munk, personal communication with TAAA 2010).
} 
Fig. 1 Schematic of the layout of the two Mulberry Harbours
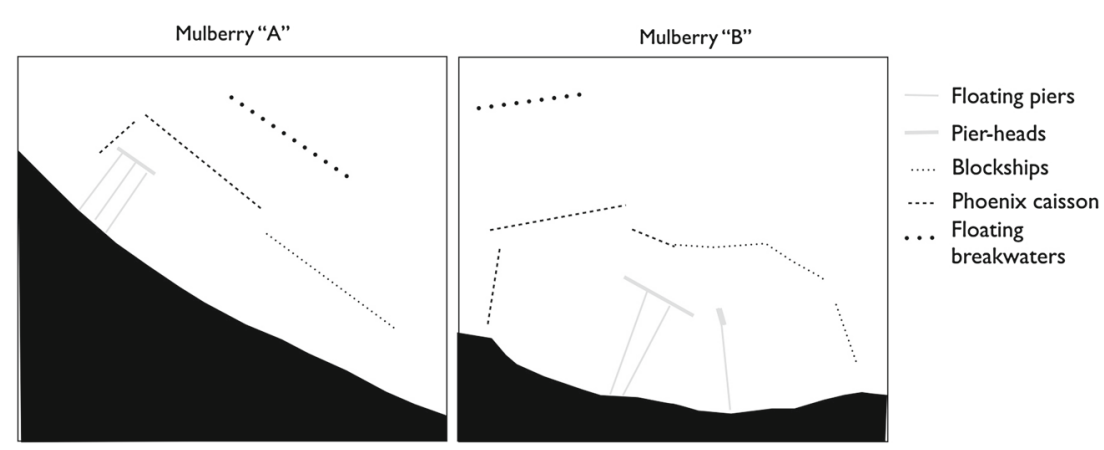

information being given in Little (1948), Harris (1989) and Adcock and Taylor (2009).

\subsection{Planning}

The initial plan for harbours started with Winston Churchill's memo to the Chief of Combined Operations from May 1942

"Piers for use on beaches.

They must float up and down with the tide. The anchor problem must be mastered. Let me have the best solution worked out. Don't argue the matter. The difficulties will argue themselves."

Several designs made it as far as being trialled before the flexible roadways suggested by Royal Engineer Allan Beckett were chosen. Only at the Quebec conference (QUADRANT) where the Allies met to discuss the invasion of Northern Europe in August 1943 was the decision taken to protect the piers with breakwaters. This left 8 months to design and construct the breakwaters. Whilst there are many aspects to the engineering of the harbours which are impressive, perhaps the most impressive is the speed at which the project was delivered.

Two harbours were constructed: Mulberry A in the American sector and Mulberry B on the British beach off the village of Arromanches. Both harbours were to be twice the size of Dover harbour, to be operational 14 days after D-Day and to have a design life of 90 days.

\subsection{Design and layout}

Schematics of the two harbours are presented in Fig. 1. Running up the beach were floating roadways ('Whales') over which transport vehicles and tanks could drive. These were designed to be compliant so that they would move with the waves and tide rather than trying to resist the motion. These connected to pierheads ('Spuds') which could be raised and lowered with the tide in a similar manner to a modern jack-up rig.

A variety of approaches were used for the breakwaters. In relatively shallow areas old ships were scuttled. In deeper water 'Phoenix' caissons were used. These were towed across the channel and sunk in place. Finally, an outer floating breakwater (the 'Bombardons') was used to give extra protection and increase the area of tranquil water.

\subsection{Deployment}

The harbours were transported to the Normandy beaches in the days after D-Day. Mulberry A was installed very quickly and ahead of schedule although various shortcuts were made in its installation, such as using fewer anchors on the roadway than specified in the design. Mulberry B was installed on time with some minor changes as the military commanders (who mostly did not have technical backgrounds) overruled the engineers and placed the breakwaters in deeper water than originally planned to increase the area of protected water.

Fourteen days after D-Day a severe storm arose hereafter referred to as 'The Storm'. The intensity of The Storm has been much speculated on and is the topic of this paper although the figure of 1 in 40 years for the return period is commonly used. The storm was severe enough to damage Mulberry A beyond repair and cause significant damage to Mulberry B. Figure 2 is a still from a video showing a Bombardon breakwater which broke loose and collided with

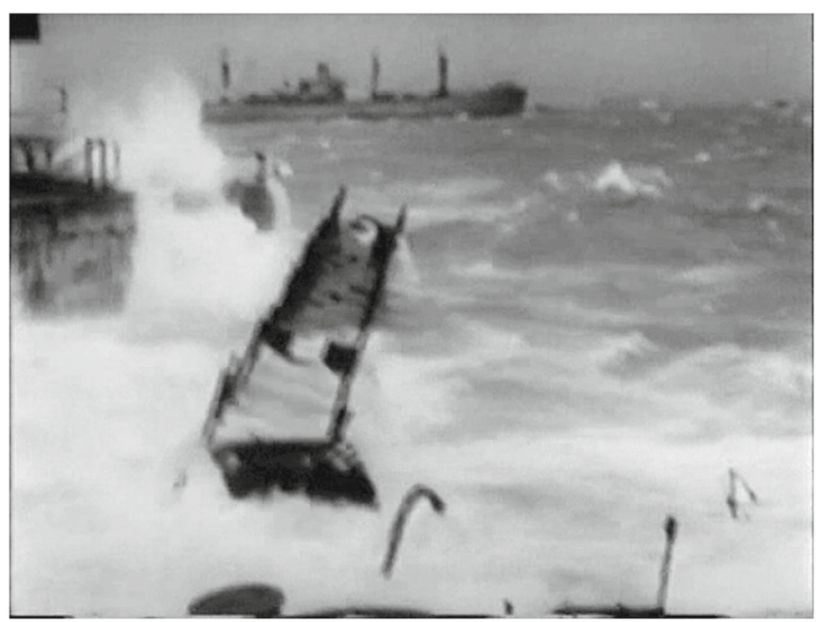

Fig. 2 A still from a video taken during the Storm showing a Bombardon loose adjacent to a Phoenix caisson 
Table 1 Design conditions used in the design of the Mulberry Harbours

\begin{tabular}{ll}
\hline Wind speed & Force 6 \\
Wave height & $2.44 \mathrm{~m}$ \\
Wave length & $36.6 \mathrm{~m}$ \\
\hline
\end{tabular}

Table 2 Different estimates for the severity of The Storm

\begin{tabular}{lll}
\hline Source & Wind & Maximum wave height (m) \\
\hline Harris (1989) & Force 6/7 & $3.65-4.26$ \\
Hartcup (1977) & Force 6/7 & $3.41-3.85$ \\
Hastings (1999) & Force 6 & - \\
Hodge (1946) & Force 9 & - \\
Jellett (1948) & Force 7 & 3.65 \\
Lochner et al. (1948) & - & 3.95 \\
McGivern (1945) & Force 7/8 & 4.57 \\
Taylor (1946) & Force 8 & - \\
\hline
\end{tabular}

the caissons. Following the storm, parts of Mulberry A were salvaged for Mulberry B which continued in operation (following reinforcements during a 'winterization') until well beyond the original design life of the harbours when the port of Antwerp was taken by the Allies.

\subsection{Design waves and severity of the storm}

The designers of the harbour were given a number of design conditions as shown in Table 1 (converted to SI units). These values are quoted by Hodge (1946) and are used in the calculations of freeboard and design loads by Jellett (1948) and Lochner et al. (1948). It is not stated how these numbers were selected. Although not stated explicitly, from the way in which these were used in calculation it is clear that these were considered to be the 'design' wave, rather than the significant wave-height.

This paper focuses on the waves in The Storm. Accounts vary as to the wind speed and wave heights. Estimates of wind speed and wave height vary from force six on the Beaufort Scale to force nine as given in Table 2. Accurate measurements would have been difficult under the pressure of war-time conditions and no details are available for any of these data.

\section{Wave hindcast}

\subsection{Methods}

The input to the modelling in this paper is taken from a dedicated study of the meteorological conditions including the waves carried out by ECMWF as part of the ERACLIM project (Simmons et al. 2014). ERA-CLIM aims at reconstructing records of atmospheric conditions over the twentieth century by applying the techniques of modern numerical weather analysis and forecasting to historical surface and upper-air observations. The ERA-CLIM system assimilates observations using a version of the ECMWF operational system run operationally in 2012-2013 albeit with a number of modifications (Dee et al. 2014). The assimilation system used a horizontal resolution of about $125 \mathrm{~km}$, but specific to the study of June 1944, forecasts were also run from the 00 UTC and 12 UTC analyses using a $16-\mathrm{km}$ resolution to enhance the realism of wind and cloud fields. This was done both for 2-7 June to cover the critical D-Day period and for 18-23 June to cover The Storm. These downscaled short-range forecasts (steps 6, 9, 12 and $18 \mathrm{~h}$ ) were then used to build a 3-hourly time history of the wind used to drive the stand-alone operational configuration of ECMWF ocean-wave model ECWAM (Bidlot 2012; ECWMF 2016) with a spatial resolution of about $11 \mathrm{~km}$ and 36 directions and 36 frequencies for the spectra, to provide reconstructions of the sea-state over these two key periods.

The ECMWF study predicted the winds and waves in the English Channel, but as with all large scale hindcasts, did not bring the waves into the very shallow-water in the near shore, nor did it consider the impact of the tides. Three-hourly outputs of this system were used to drive the offshore boundary of the near shore model

In this study, we use SWAN (Booij et al. 1997) to propagate the hindcast into the nearshore region. At the offshore boundary, we force the model using significant wave-height, $H_{\mathrm{s}}$, peak period, $T_{\mathrm{p}}$, and mean wave direction. We assume a JONSWAP spectrum (see Tucker and Pitt 2001). We use the third generation SWAN model with wind growth of waves, whitecapping, depth-induced breaking, triads and quadruplet wave-wave interactions, bottom friction and wave-induced set-up. We use SWAN default values suggested by HR Wallingford for all empirical coefficients. The wind is taken from the ECWMF model. We run the model in steady-state mode for each $3 \mathrm{~h}$ interval from the hindcast. Given the size of the domain and the variations in wind and tide this is a reasonable assumption. We consider the influence of tidal currents in the region using data taken from the model of Adcock and Draper (2014). Preliminary calculations found that the currents had only a minor influence on the predicted waves and so currents are not included in our analysis any further. However, water level had a significant impact on the wave-height. Tidal amplitude data for the port of Arromanches was taken from relevant stations in the Admiralty TotalTide software.

We use rectangular nested computational grids as shown in Fig. 3. The outer grid uses a resolution of $500 \mathrm{~m}$ and the inner grids a resolution of $250 \mathrm{~m}$. We do not include the presence of the harbours in the model and assume that there has been no significant change to the bathymetry in the 70 years since the D-Day landings. Examination of nautical charts and other data over time show no obvious major changes. Further details of the modelling are given by Jackson (2016). 
Fig. 3 The domains used for this study-outer domain with thick lines and inner with thin lines. Outer domain has width of $140 \mathrm{~km}$. Approximate contours shown for 20 and $40 \mathrm{~m}$ water depth. Map taken from Openstreetmap

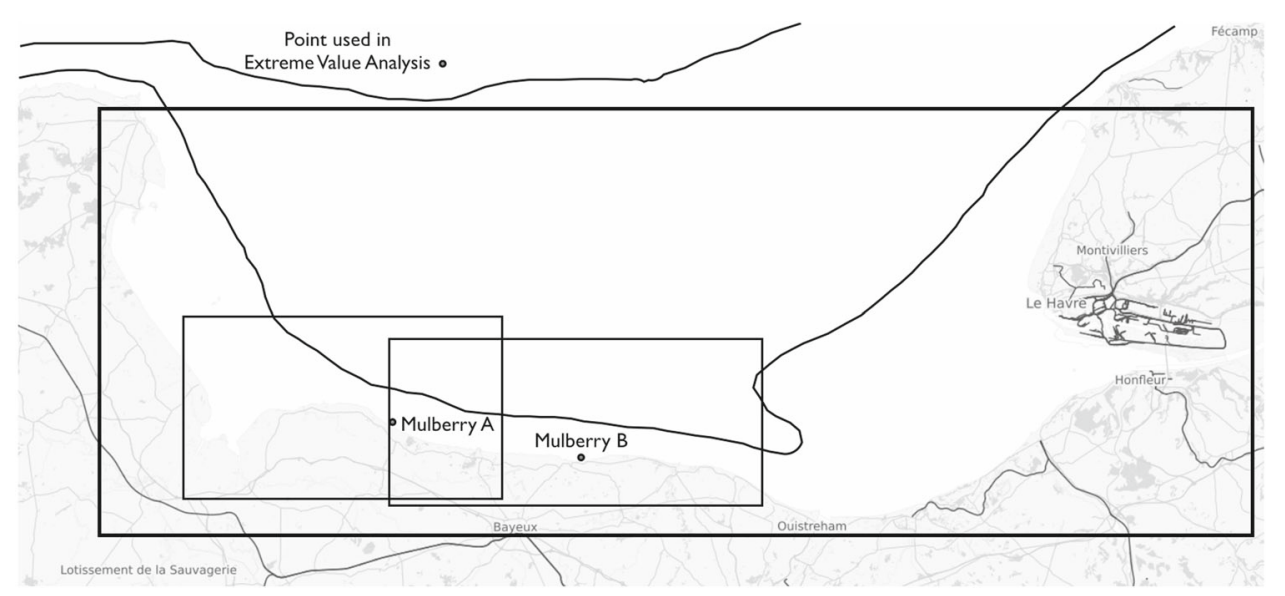

\subsection{Results}

We present the results in a series of figures. Figures 4 and 5 present contours of the predicted significant wave height at Mulberry A and Mulberry B at the peak of The Storm. We also extract time series from these figures of significant wave height for the locations shown (A1, A2, A3, B1, B2, B3). These are shown in Figs. 6 and 7.

The results show The Storm building up over 19th June and reaching a peak during the night of the 20th. The influence of the tide can clearly be seen, with more wave energy being dissipated at low water (there is only a small difference in high tide heights between the harbours). There is a significant difference between the sea-states between the two harbours. At the offshore points, A1 and B1, the wave heights are similar but reduce more at Mulberry B than at Mulberry A as waves approach the breakwaters. At $\mathrm{A}$, the $-20 \mathrm{~m}$ (Chart Datum) contour is closer to shore and the waves are approaching close to the beach normal (approximately $10^{\circ}$ ), so there is only a small reduction due to refraction in wave height from $\mathrm{A} 1$ to $\mathrm{A} 2$ and $\mathrm{A} 3$ (Figs. $4,6)$. At $B 1$, the waves are approaching from about $35^{\circ}$ to the beach normal leading to greater reduction in wave height due to refraction. There are also banks immediately northeast of the harbour that dissipate and divert some of the wave energy.

Our simulations suggest that, at the harbours, Mulberry A encountered significantly larger waves than Mulberry B. This helps explain why this harbour failed whilst Mulberry $B$ largely survived. There is a significant reduction in wave height moving inshore to Mulberry B-in contrast to the larger and almost uniform wave height across the different locations for Mulberry A. These results suggest that there is little refractive focusing of energy at low tide at the site of the Mulberry 'B' Bombardons as suggested in the more simplistic modelling undertaken by Adcock and Taylor (2009).

It is interesting to compare our estimates with those in the literature. Table 2 presents 'Maximum waveheights' quoted by different sources. In all cases, we also have no information as to where the waves were measured although in most cases the value given is likely to refer to Mulberry $\mathrm{B}$. There is ambiguity between different sources as to exactly what is meant by maximum wave height—does it mean the largest significant wave height, $H_{\mathrm{s}}$, [which would have been defined as the average of the third largest waves at the time (see discussion in Tucker and Pitt 2001)] or the largest wave observed? The latter is perhaps more likely but we cannot be certain. To estimate maximum wave height from significant wave height by simply multiplying by 1.8 is an approximation which is often used. As such our results appear to be consistent with observations.

We believe that the model presented here represents the best estimate that can be made for the wave conditions during The Storm. However, the accuracy of these results must, of course, be treated with caution. We have not been able to find measured data to validate our SWAN model and generally such models can be improved by adjusting empirical parameters so that these are site-specific. Further, the SWAN model is dependent on the input from the hindcast. Whilst this is the best available, it is obviously based on the inevitably patchy data recorded during the period in question. However, even if there is some uncertainty in the predicted wave heights, we can conclude that the sea-state was significantly more severe at Mulberry A than at Mulberry B (1.3 times higher in our model), which to our knowledge is a new result.

\section{Return period}

We briefly examine the sea-state statistics for the area. We analyse sea-state data at a location $0.77 \mathrm{~W} 49.75 \mathrm{~N}$ on the outer edge of the domain and exposed to seas from the west. We take our data from a wave model using ERA-interim 6 hourly wind fields (1979-2014), with the operational cycle CY41R1 (see ECWMF 2016), configured on the $28 \mathrm{~km}$ global grid, with 36 directions and 30 frequencies for the spectra. This 
Fig. 4 Significant wave height field from SWAN at the peak of the storm at 21:00 20/06/1944.

Also, shown are the approximate locations of the breakwaters and jetties that formed Mulberry Harbour A at Omaha Beach

Fig. 5 Significant wave height field from SWAN at the peak of the storm at 21:00 20/06/1944.

Also, shown are the approximate locations of the breakwaters and jetties that formed Mulberry Harbour B at Arromanches
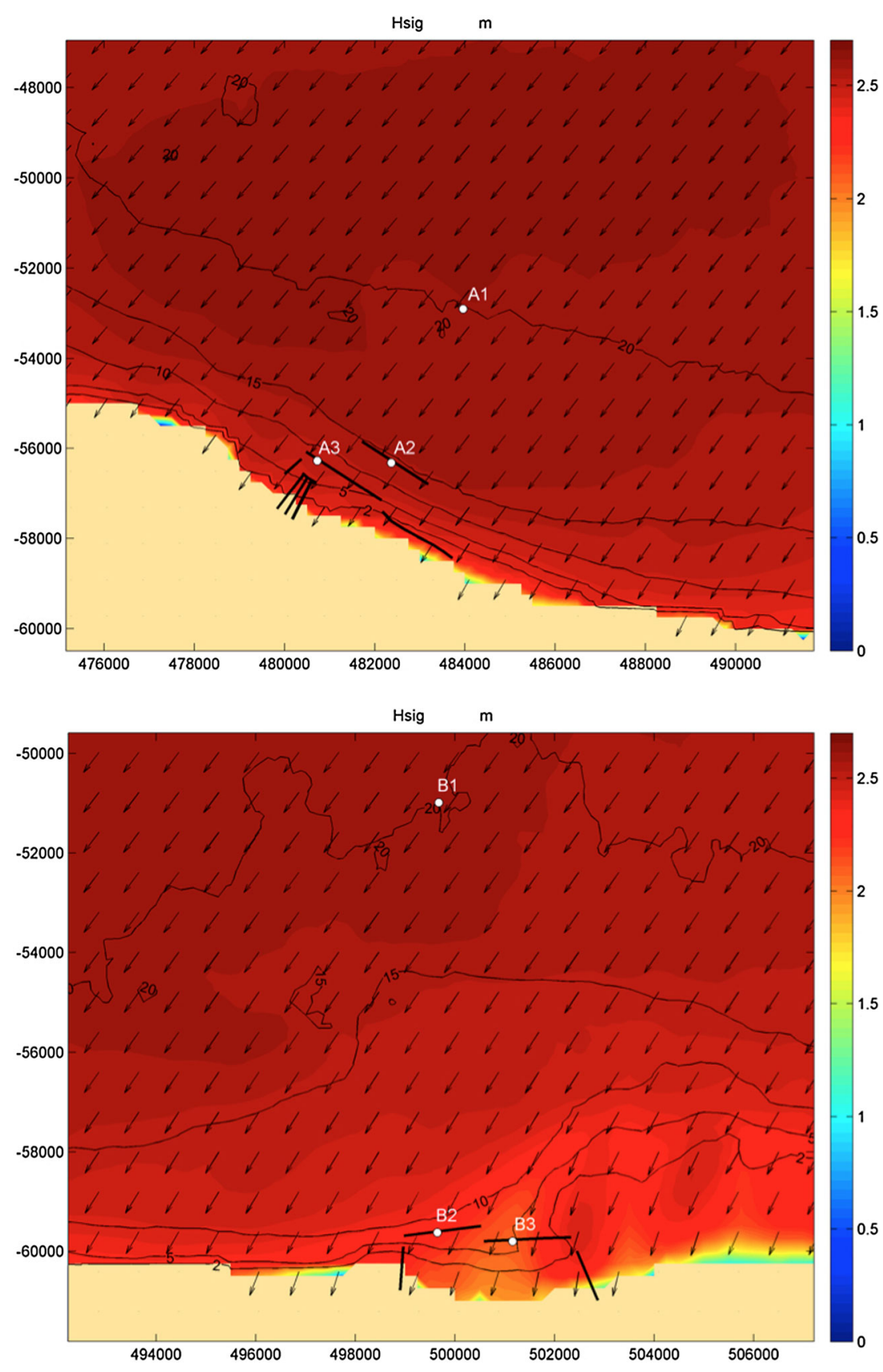

global hindcast was produced in preparation of ECMWF next reanalysis (ERA5). The effect of water level change and surface current due to tides is neglected at this deep water location. The output used is hourly. Thus, we have 36 years of model data to analyse.

We start by considering the omni-directional extremes. As the Mulberry Harbour sites are sheltered from the most severe storms (coming from the West), this is most relevant to the transportation during D-Day itself. At this location, the hindcast for D-Day (6 June) suggests the waves had a significant wave height of $1 \mathrm{~m}$. Taking all the data for the months of June for the 36 years available this wave height would have been exceeded $19 \%$ of the time. If instead the invasion had been planned for May it would have been exceeded $26 \%$ of the time. Although we can only speculate how Eisenhower might have acted given slightly different weather conditions or predictions, it is generally considered that the decision to go ahead, given the weather, was a marginal one. Given these data, it is clear there was quite a high chance that the invasion would have had to have been postponed.

We now examine the waves that might have been expected at the Mulberry Harbours. To do this, we use the local SWAN model to transform the long-term ECMWF reanalysis to the nearshore. Running the model for every hourly time- 
Fig. 6 Significant wave height at locations around Mulberry A

Fig. 7 Significant wave height at locations around Mulberry
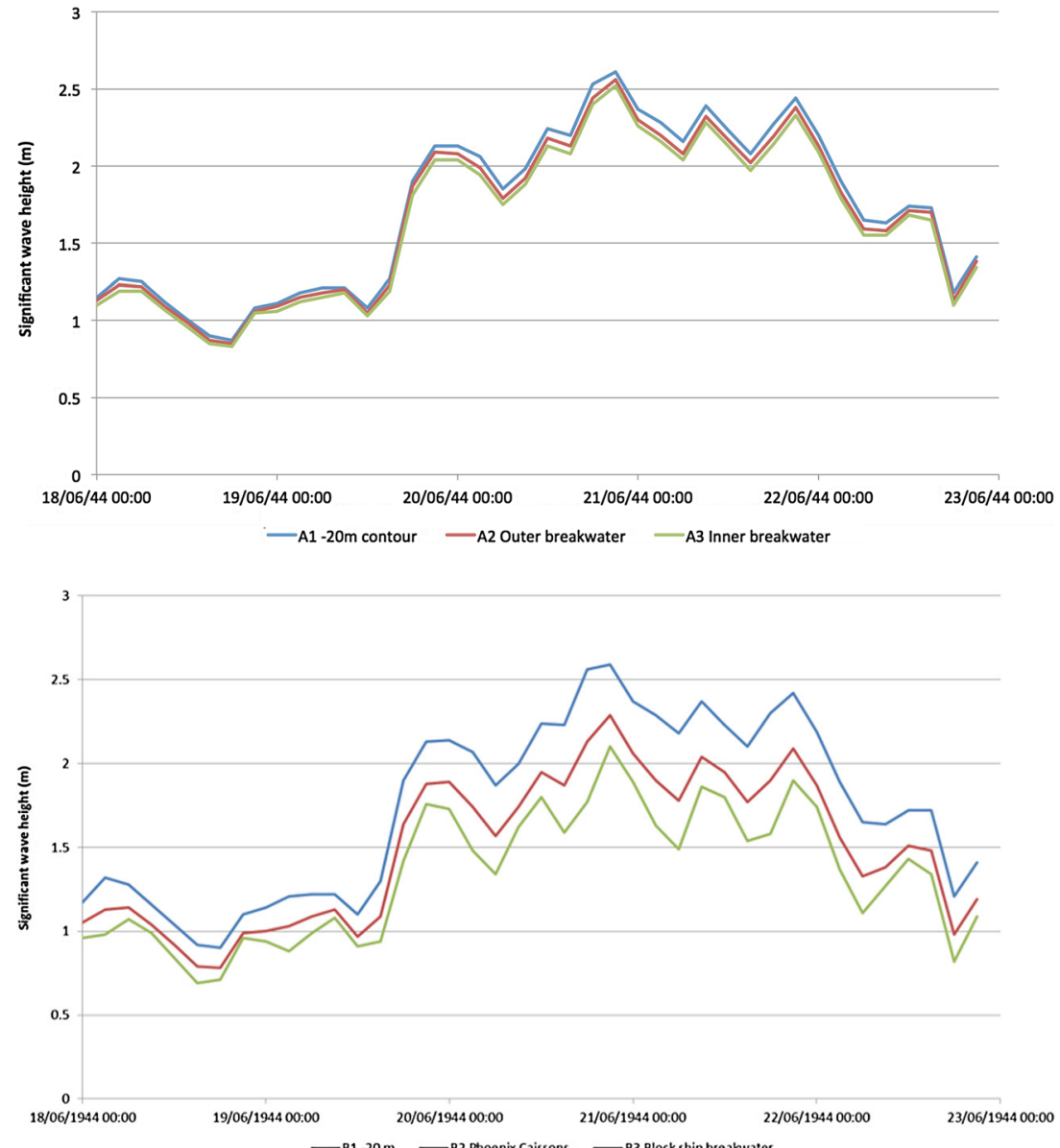

step interval within the 36-year time series would be very computationally expensive. Therefore, the time series was simulated using an emulator, where a number of wind, wave and tidal water level conditions, representing the full range of the climate, are selected and modelled using SWAN, then the full time series is simulated by applying a transform function derived from the results of the representative model runs (Camus et al. 2011a, b; Gouldby et al. 2014).

Using this approach, a time series of wave conditions at the nearshore points (A1, A2, A3, B1, B2 and B3) were produced that were then analysed and compared to the predictions during the Storm. Extreme value analysis was performed on the long-term 1979-2014 hindcast, both for the summer months of June, July and August and also for the full year. Large significant wave height events were selected from the time series using a peaks-over-threshold approach (thresholds of $H_{\mathrm{s}}=1.58 \mathrm{~m}$ for $\mathrm{A} 1$ and $H_{\mathrm{s}}=1.72 \mathrm{~m}$ for B1 were used). The peak events were then fitted with a generalised Pareto distribution (GPD).

The results from this analysis, considering the summer months of June, July and August are presented in Fig. 8 for point A1 and in Fig. 9 for point B1. In each figure, the events from the long-term hindcast are shown as black points and the best fit GPD distribution shown as the solid line. The dashed lines show $95 \%$ confidence limits to the fitted distribution. The plotting position of the events was selected following Beard (1943). The horizontal dotted line shows the significant wave height during The Storm of June 1944.

Based on this approach, the return period for The Storm was estimated as between 40 and 50 years at B1 (offshore from Mulberry B) and at approximately 80 years at A1 (offshore from Mulberry A), considering summer months only. The difference between the predicted return periods offshore from the two harbours is because the largest events in the 1979-2014 hindcast were predicted to have smaller wave heights at A1 than at B1, possibly because A1 is more sheltered from the west, the prevailing storm direction, by the Cherbourg peninsula.

In the context of annual storm wave conditions, wave heights during The Storm of June 1944 were not so unusual. Following a similar approach, the annual return period was predicted to be 0.33 year at A1 and 0.17 year at B1. This is of interest considering that Mulberry B, which largely survived the storm, despite its intended design life of 3 months, remained operational for 10 months after D-Day following a programme of 'winterization'. 
Fig. 8 Extreme wave Mulberry Harbour A based on ECMWF wave hindcast 1979-2014 and nearshore SWAN model. The horizontal dotted line shows the predicted wave height at the peak of the 1944 storm predictions at A1 offshore from

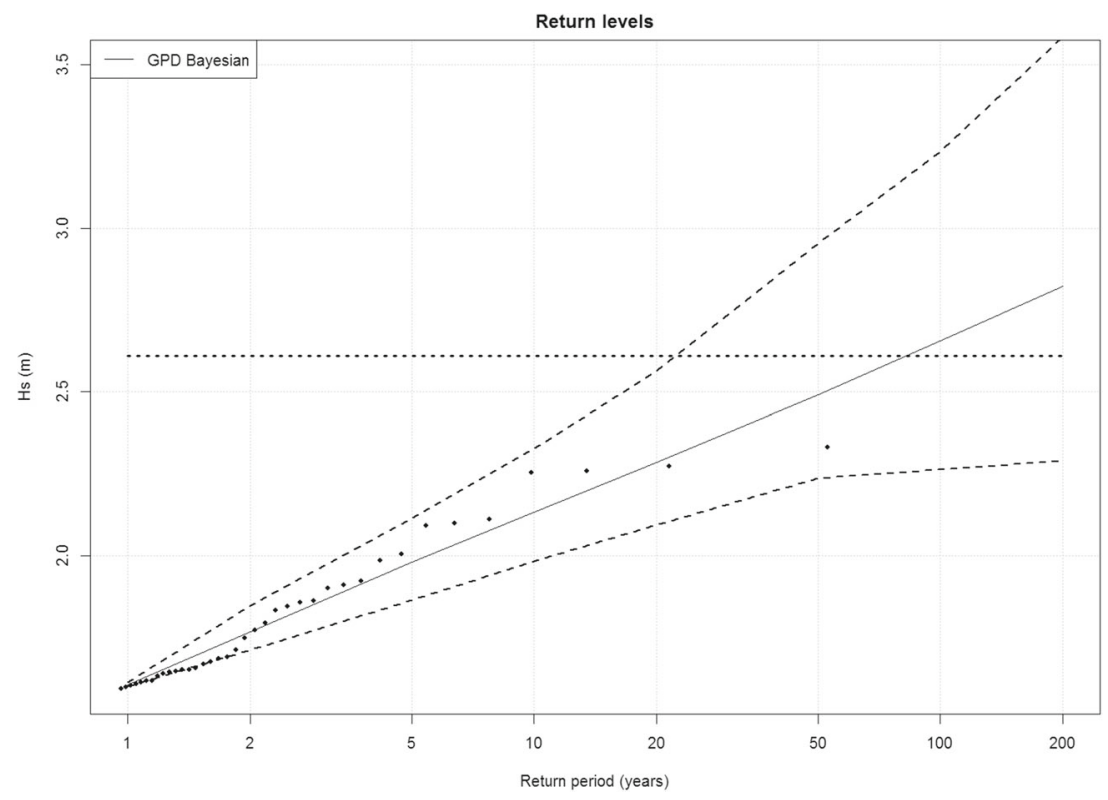

Fig. 9 Extreme wave predictions at $\mathrm{B} 1$ offshore from Mulberry Harbour B based on ECMWF wave hindcast 1979-2014 and nearshore SWAN model. The horizontal dotted line shows the predicted wave height at the peak of the 1944 storm

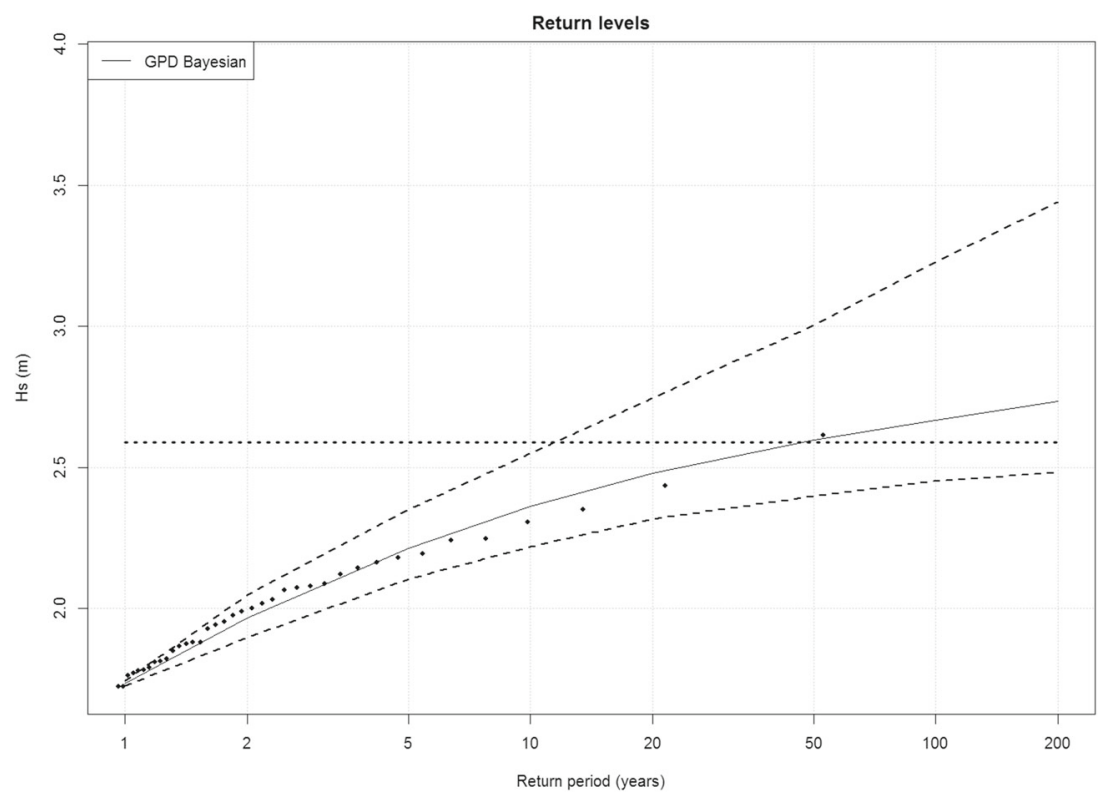

\section{Conclusions}

In this paper, we have looked at the metocean conditions experienced by the Mulberry Harbours during their deployment during the Second World War. We have carried out a study of the waves experienced by the harbours during the severe storm that occurred a fortnight after D-Day. This suggests that the American Mulberry experienced a significant wave height of $2.5 \mathrm{~m}$ with the British Mulberry being hit by an $H_{\mathrm{s}}$ of $2.1 \mathrm{~m}$. With loads increasing faster than linear in wave height this may partially explain why the British Mulberry largely survived the storm whilst the American one was destroyed.
We have also considered the return period of the storm which hit the harbours. The harbours were certainly unlucky to experience a storm of this severity over the summer months. The storm is usually described as a 1 in 40 years event. This estimate is consistent with our findings. The wave height for which the harbours were designed was probably too small - the design conditions would have been exceeded in the majority of summers. General Eisenhower and the Allies were unfortunate to have the Storm hit when it did. However, the survival of Mulberry B and its subsequent operation for 10 months was a stroke of great good fortune for the Allies, and a tribute to the designers, construction workers and the brave men who manned it. 
Acknowledgements We thank Andrea Schnabl (Oxford) for providing the tidal currents for this modelling. We thank HR Wallingford for their support with this project. We thank ECMWF for the hindcast data without which this work would not have been possible.

Open Access This article is distributed under the terms of the Creative Commons Attribution 4.0 International License (http://creativecomm ons.org/licenses/by/4.0/), which permits unrestricted use, distribution, and reproduction in any medium, provided you give appropriate credit to the original author(s) and the source, provide a link to the Creative Commons license, and indicate if changes were made.

\section{References}

Adcock TAA, Draper S (2014) On the tidal stream resource of two headland sites in the English Channel: Portland Bill and Isle of Wight. In: ASME 2014 33rd international conference on ocean, offshore and arctic engineering, San Francisco, California, USA, 8-13 June 2014. American Society of Mechanical Engineers

Adcock TAA, Taylor PH (2009) The Mulberry Harbours: a review of an early example of offshore engineering. In: ASME 2009 28th international conference on ocean, offshore and arctic engineering, American Society of Mechanical Engineers, pp 567-574

Beard LR (1943) Statistical analysis in hydrology. Trans Am Soc Civ Eng 108(1):1110-1121

Bidlot JR (2012) Present status of wave forecasting at ECMWF. In: Workshop on ocean waves, pp 25-27

Booij N, Holthuijsen LH, Ris RC (1997) The "SWAN" wave model for shallow water. Coast Eng Proc 1(25):668-676

Camus P, Mendez FJ, Medina R (2011a) A hybrid efficient method to downscale wave climate to coastal areas. Coast Eng 58(9):851-862

Camus P, Mendez FJ, Medina R, Cofiño AS (2011b) Analysis of clustering and selection algorithms for the study of multivariate wave climate. Coast Eng 58(6):453-462

Dean RG, Dalrymple RA (1991) Water wave mechanics for engineers and scientists. World Scientific, Singapore
Dee DP, Balmaseda M, Balsamo G, Engelen R, Simmons AJ, Thépaut JN (2014) Toward a consistent reanalysis of the climate system. Bull Am Meteorol Soc 95(8):1235-1248

ECWMF (2016) European Centre for Medium Range Weather Forecasts. Tech. rep, IFS Documentation

Gouldby B, Méndez F, Guanche Y, Rueda A, Mínguez R (2014) A methodology for deriving extreme nearshore sea conditions for structural design and flood risk analysis. Coast Eng 88:15-26

Harris A (1989) The Mulberry Harbours. Trans Newcom Soc 61(1):8199

Hartcup G (1977) Code name Mulberry: the planning, building, and operation of the Normandy harbours. David \& Charles, New York

Hastings M (1999) Overlord: D-day and the Battle for Normandy 1944. Pan Macmillan, Oxford

Hodge W (1946) The Mulberry Invasion Harbours-Their design, preparation and installation. Struct Eng 24(3):125-192

Jackson Z (2016) How ocean waves almost wrecked the D-Day landings. University of Oxford, Oxford, Final Year Project

Jellett JH (1948) The lay-out, assembly, and behaviour of the breakwaters at Arromanches Harbour (Mulberry B). In: The civil engineer in war, vol 2. Institute of Civil Engineers, London

Little DH (1948) Discussion on Mulberry Components. The civil engineer in war. A symposium of papers on war-time engineering problems, vol 2

Lochner R, Faber O, Penny WG (1948) The 'Bombardon' floating breakwater. In: The civil engineer in war, vol 2, Institute of Civil Engineers, London

McGivern C (1945) Harbour called 'Mulberry'. Pendulum Publications

Reeve D, Chadwick A, Fleming C (2004) Coastal engineering: processes, theory and design practice. CRC Press, Boca Raton

Simmons S, Hersbach H, Poli P, Bidlot J, Dee D (2014) ECMWF revisits the meteorology of the D-Day period. ECMWF Newslett 140:7

Taylor JE (1946) The last passage. George Allen \& Unwin, London

Tucker MJ, Pitt EG (2001) Waves in ocean engineering, vol 5. Elsevier, Kiddlington 